\title{
Pre-Processing Steps for Segmentation of Retinal Blood Vessels
}

\author{
V.P. Patil \\ Assistant Professor, \\ Department of Electronics and Telecommunication \\ Engg \\ R.C.Patel Institute of Technology, Shirpur-425 405 \\ Dist-Dhule, Maharashtra, India
}

\begin{abstract}
Segmentation of blood vessels in retinal images is an important part in retinal image analysis for diagnosis and treatment of eye diseases for large screening systems. In this paper, we addressed the problem of background and noise extraction from retinal images. Blood vessels usually have central light reflex and poor local contrast, hence the results yield by blood vessel segmentation algorithms are not satisfactory. We used different preprocessing steps which includes central light reflex removal, background homogenization and vessel enhancement to make retinal image noise-free for post-processing. We used mean and Gaussian filtering along with Top-Hat transformation for noise extraction. The preprocessing steps were applied on 40 retinal images of DRIVE database available publically. Results show the darker retinal structures like blood vessels, fovea, and possible presence of microaneurysms or hemorrhages, get enhanced as compared to original retinal image and the brighter structures like optic disc and possible presence of exudates were get removed. The presented technique will definitely improve automatic fundus images analysis also be very useful to eye specialists in their visual examination of retina.
\end{abstract}

\section{General Terms}

Pattern Recognition, Segmentation, Blood Vessels.

\section{Keywords}

Blood Vessel Segmentation, Noise Extraction, DRIVE Database, Top-Hat Transformation.

\section{INTRODUCTION}

Retinal images are used in the diagnoses of eye diseases by ophthalmologist. Retina images of human are taken using fundus camera, then analyzing retina images using image processing and pattern recognition techniques to make specific diagnoses decision. Color fundus image forms the basis for manual assessment in screening. Such manual assessment however is not scalable in large-scale screening scenario, particularly in developing countries like India either due to the scarcity of skilled manpower or unavailability of high end imaging equipment at the point of care. For such large-scale screening we need automated image processing algorithms for assessment and detection of eye disease.

The image processing and pattern recognition of fundus image includes image enhancement, image restoration, and image segmentation. The identification of fundal landmark features such as the optic disc, fovea and the retinal vessels as reference co-ordinates is a prerequisite before systems can achieve more complex tasks identifying pathological entities. In retinal image, generally retinal structures optic disc and

\author{
P. R. Wankhede \\ Assistant Professor, \\ Department of Electronics and Telecommunication \\ Engg \\ Shri Sant Gajanan Maharaj College of Engineering, \\ Shegaon-444203
}

presence of exudates or reflection artifacts are brighter while blood vessels, fovea and possible presence of microaneurysms or hemorrhages look darker. Fig. 1 shows color fundus retina image with its anatomical structures optic disc, fovea and blood vessels.

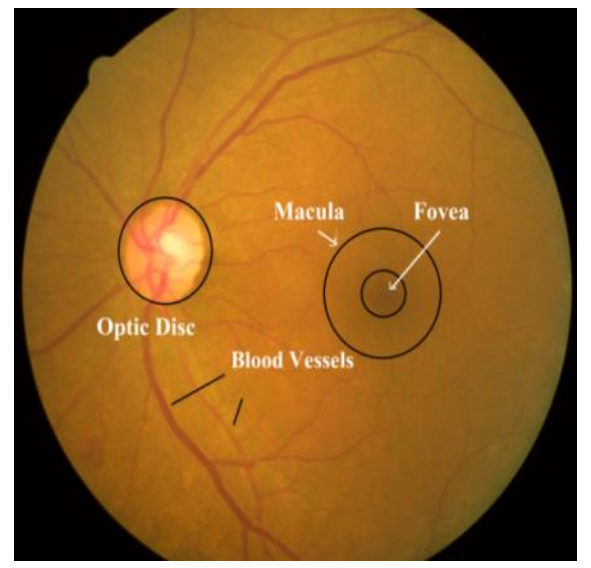

Fig 1: Color Fundus image with anatomical structures

Blood vessels act as a landmark for localizing the retinal structures like optic disc (OD), fovea and hard exudates (HE) (disease lesions). The blood vessels can have measurable abnormalities in diameter, color and tortuosity due to systemic or local ocular disease. For example, central retinal artery occlusion usually causes generalized constriction of retinal arteries, Hypertension may result in focal constriction of retinal arteries, central retinal vein occlusion typically produces dilated tortuous veins, arteriosclerosis can cause the arteries to acquire a copper or silver color, and diabetes can generate new blood vessels (neovasularization). Thus, a reliable method of vessel detection is needed, which preserves various measurements [3].

Since retinal images show some lighting variations, poor contrast and noise, it is difficult to segment and detect the exact blood vessels. It needs to remove these effects from retinal image which help in better segmentation and detection of blood vessels and fovea.

The paper is divided in four sections. Section one describes about need of blood vessels segmentation and the problems associated with that. Section two describes the details of dataset used; Section three detailed with different preprocessing steps such as central light reflex removal, background homogenization and blood vessel enhancement. The results are detailed in section four followed by conclusion and references. 


\section{MATERIALS}

A few public datasets are available for comparison and evaluation of automatic detection methods; such as DRIVE database (2004) which is used for comparative studies on segmentation of blood vessels in retinal images. The DRIVE database comprises 40 eye-fundus color images (seven of which present pathology) taken with a Canon CR5 nonmydriatic 3CCD camera with a 45 field-of-view (FOV). Each image was captured at $768 \times 584$ pixels, 8 bits per color plane and in spite of being offered in LZW compressed TIFF format, and they were originally saved in JPEG format. The database is divided into two sets: a test set and a training set, each of them containing 20 images. The test set provides the corresponding FOV masks for the images, which are circular (approximated diameter of 540 pixels) and two manual segmentations generated by two different specialists for each image. The selection of the first observer is accepted as ground truth and used for algorithm performance evaluation in literature. The training set also includes the FOV masks for the images and a set of manual segmentations made by the first observer and second observer.

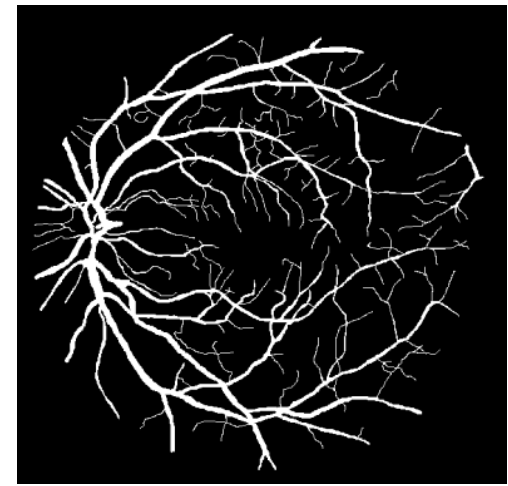

Fig 2: Manual blood vessel segmentation of DRIVE database retinal image considered as ground truth

\section{PREPROCESSING OF RETINAL IMAGES}

Color fundus retinal images show some lighting variations, poor contrast and noise. To reduce these imperfections and generate images more suitable for extracting blood vessels, need to understand the properties of fundus image then apply preprocessing steps.

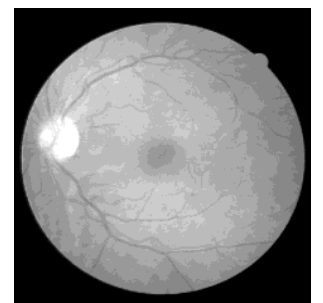

(a)

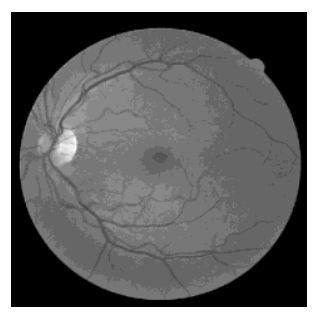

(b)

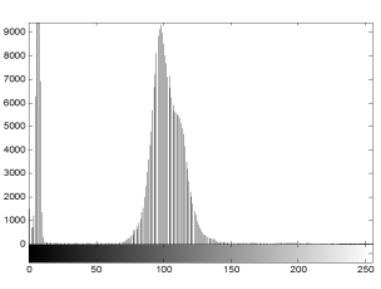

(d)

(e)

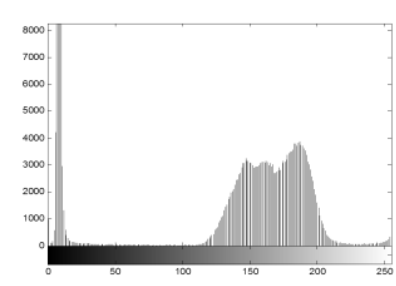

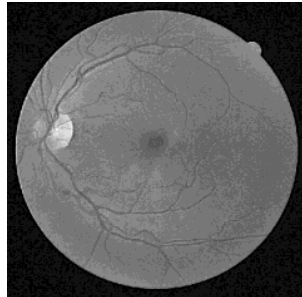

(c)

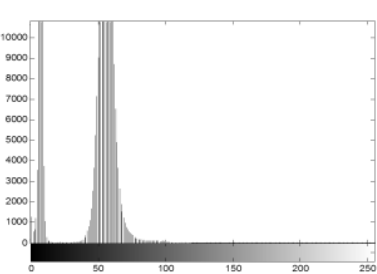

(f)
Fig 3: (a) Red Plane of Retina Image (b) Green Plane of Retina Image (c) Blue Plane of Retina Image (d), (e) and (f) Histogram of Red plane, Green Plane and Blue Plane respectively

\section{Properties of blood vessels:}

a. The blood vessels usually have small curvature, the anti-parallel pairs may approximated by piecewise linear segments.

b. Vessels have lower reflectance than other retinal surfaces, so they appear darker relative to the background.

c. Vessel size may decrease when moving away from the optic disk, the width of a retina vessel may lie within the range of $2-10$ pixels.

d. The intensity profile varies by a small amount from vessel to vessel.

e. The intensity profile has a Gaussian shape.

By understanding and studying above properties of blood vessels we need to apply following preprocessing steps, so that blood vessels easily get detected and segmented for further major post-processing steps [2].

a. Vessel central light reflex removal,

b. Background homogenization, and

c. Vessel enhancement.

Following sub point shows a description of above preprocessing steps applied to a DRIVE database fundus images along with their result.

\subsection{Vessel Central Light Reflex Removal}

Blood vessels have lower reflectance than other retinal surfaces, so they appear darker relative to the background. Although the typical vessel cross-sectional gray-level profile can be approximated by a Gaussian shaped curve i.e. inner vessel pixels are darker than the outermost vessel pixels, some blood vessels include a light streak (known as a light reflex) which runs down the central length of the blood vessel [2].

The green plane of the fundus image is used to remove this brighter strip. Then it is filtered by applying a morphological opening using a three-pixel diameter disc as structuring element. Fig 3 shows the green plane of fundus image and their histograms. The green channel shows the background gray levels are higher than that of vessels gray levels.

\subsection{Background Homogenization}

A background intensity variation due to non-uniform illumination is associated with retinal fundus images. Consequently, background pixels may have different intensity for the same image and, although their gray-levels are usually higher than those of vessel pixels as given in green channel images, so the intensity values of some background pixels is comparable to that of brighter vessel pixels. For the purpose of removing these background lightening variations, a shade- 
corrected image estimated from a background. Shade corrected image is the result of a filtering operation with a large arithmetic mean kernel. For this purpose, we have used the steps given in [2] and are described as it is below. Firstly, a mean filter is applied to smooth occasional salt-and-pepper noise. Further noise smoothing is performed by convolving the resultant image with a Gaussian kernel of dimensions $\mathbf{m} \times \mathbf{n}=\mathbf{9} \times \mathbf{9}$, mean $\boldsymbol{\mu}=\mathbf{0}$ and variance $\boldsymbol{\sigma}=\mathbf{1 . 8}$. The Gaussian kernel is defined as:

$$
\begin{aligned}
& h_{g}(m, n)=e^{\frac{-\left(m^{2}+n^{2}\right)}{2 \sigma^{2}}} \\
& h(m, n)=\frac{h_{g}(m, n)}{\sum_{i=1}^{n} \sum_{j=1}^{m} h_{g}(m, n)} \\
& I_{n s}=\operatorname{conv}\left(I_{\text {orginal }}, h\right)
\end{aligned}
$$

Secondly, a background image $\mathrm{I}_{\mathrm{B}}$, is produced by applying a 69x69 mean filter. Then, the difference image $I_{D}$ between $\mathrm{I}_{\mathrm{ns}}$ and $\mathrm{I}_{\mathrm{B}}$ is calculated for every pixel

$$
\mathrm{I}_{\mathrm{D}}=\mathrm{I}_{\mathrm{ns}}-\mathrm{I}_{\mathrm{B}}
$$

Finally, a shade-corrected image $\mathrm{I}_{\mathrm{SC}}$ is obtained by transforming linear intensity values into integers covering the whole range of possible gray-levels 0-255. The shadecorrected image I $_{\mathrm{SC}}$ reduces background intensity variations and enhances contrast in relation to the original green channel image.

Besides the background intensity variations in images, intensities can reveal significant variations between images due to different illumination conditions in the acquisition process. In order to reduce this influence, a homogenized image $I_{H}$ is produced as follows: the histogram of $I_{S C}$ is displaced toward the middle of the gray-scale by modifying pixel intensities according to the following gray-level global transformation function:

$$
g_{\text {out }}=\left\{\begin{array}{cl}
0, & \text { if } g<0 \\
255, & \text { if } g>255 \\
g, & \text { otherwise }
\end{array}\right.
$$

Where,

$$
\mathrm{g}=\mathrm{g}_{\text {in }}+128-\mathrm{g}_{\text {in_max }}
$$

and $g_{\text {in }}$ and $g_{\text {out }}$ are the gray-level variables of input image $I_{S C}$ and output image $I_{H}$. The variable denoted by $\mathrm{g}_{\text {in_max }}$ defines the highest gray-level of pixel in $\mathrm{I}_{\mathrm{SC}}$. By means of this operation, pixels with gray-levelg in max $_{\text {max }}$ which are observed to correspond to the background of the retina,

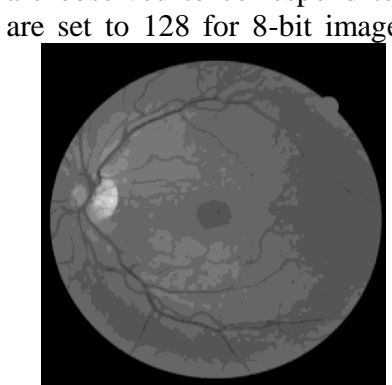

(c)

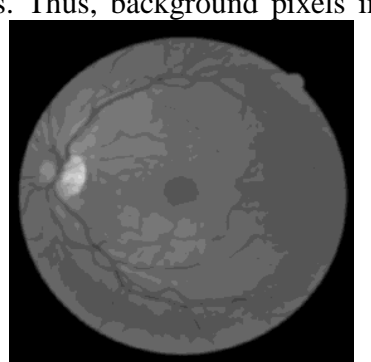

(d) images with different illumination conditions will standardize their intensity around this value.

\subsection{Vessel Enhancement}

The final preprocessing step consists on generating a new vessel-enhanced image, which proves more suitable for further post-processing steps required for segmentation and detection of blood vessels. Vessel enhancement is performed by estimating the complementary image of the homogenized image, and subsequently applying the morphological Top-Hat transformation where is a morphological opening operation using a disc of eight pixels in radius. Thus, while bright retinal structures are removed (i.e., optic disc, possible presence of exudates or reflection artifacts), the darker structures remaining after the opening operation become enhanced (i.e., blood vessels, fovea, possible presence of microaneurysms or hemorrhages). The top-hat transformation is given by

$$
\mathrm{I}_{\mathrm{TH}}=\mathrm{I}_{\mathrm{H}}^{\mathrm{C}}-\gamma\left(\mathrm{I}_{\mathrm{H}}^{\mathrm{C}}\right)
$$

where $\mathrm{IH}$ is the homogenized image and $\mathrm{I}_{\mathrm{H}}^{\mathrm{C}}$ complement of homogenized image while $\gamma$ denotes the opening operation using disk of eight pixels.

\section{RESULTS}

To get the performance of preprocessing for noise removal and background extraction, we have applied above preprocessing steps on DRIVE database of 40 fundus retinal images. Here we have shown the results for one retinal image. The results show that the preprocessed image considerably enhanced. Fig shows bright retinal structures (i.e., optic disc, possible presence of exudates or reflection artifacts) are removed while, the darker structures (i.e., blood vessels, fovea, and possible presence of microaneurysms or hemorrhages) are as it is which needs to be segment and detect for diagnosis of eye disease using retinal image processing.

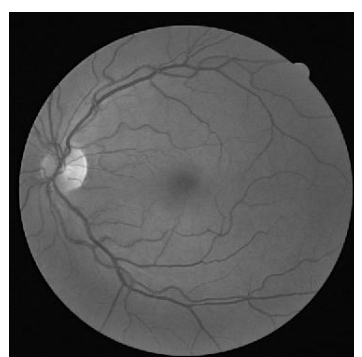

(a)

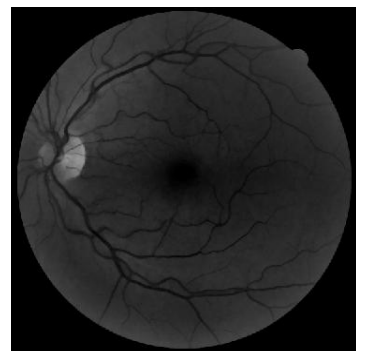

(e)

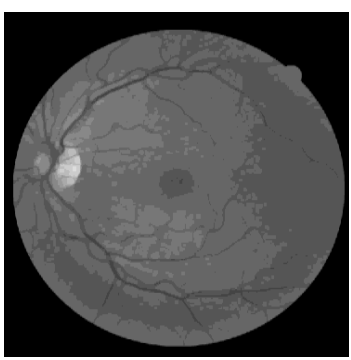

(b)

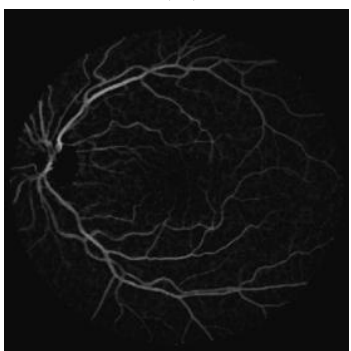

(f)
Fig 4: Preprocessing results (a) Green Channel of retinal image (b) Result of average filter (c) Result of Gaussian Filtering (d) Result of mean filter $m \times n=69 \times 69$ (e) Homogenized Image using Top-Hat transformation (f) Vessel Enhanced image 


\section{CONCLUSION}

In this paper, we have implemented the preprocessing steps for retinal image analysis. We addressed two problems of preprocessing of retinal images as noise removal and background extraction. Operations like average and Gaussian filtering, morphological operations and Top-Hat transformation were used for central light reflex removal, background extraction and blood vessels enhancement on retinal images of publicly available DRIVE database.

The preprocessing steps were simulated using MATLAB.

The results show enhanced image in which bright structures of retinal image were removed while darker structures like blood vessels, fovea etc were preserved. The preprocessed retinal image can be used for further post-processing. The results will help ophthalmologist for better diagnosis and treatment of eye diseases.

\section{REFERENCES}

[1] Muhammad Moazam Fraz et al, "An Ensemble classification-based approach applied to blood vessel segmentation", IEEE Transactions on Biomedical Engineering, Vol. 59, No. 9, September 2012, pp 25382548.
[2] Arturo Aquino et al, " A new supervised Method for blood vessel segmentation in retinal images by using gray level and moment invariants-based features", IEEE Transactions on Medical Imaging, Vol. 30, No. 1 January 2011, pp 146-158.

[3] Niall Patton et al, " Retinal Image Analysis: Concepts, Applications and Potential”, Progress in retinal in Retinal and Eye Research 25 (2006) 99-127.

[4] Kenneth W Tobin et al, "Detection of Anatomic Structures in Human Retinal Imagery", IEEE Transactions on Medical Imaging, Vol. 26, No. 12 December 2007 , pp 1729-1739.

[5] Delia Cabrera DeBuc, "A Review of Algorithms for Segmentation of Retinal Image Data Using Optical Coherence Tomography", Chapter 2, Image Segmentation, pp15-54, available at www.intechopen.com

[6] Marco Foracchia, "Luminosity and contrast normalization in retinal images", Medical Image Analysis 9 (2005) 179-190. 